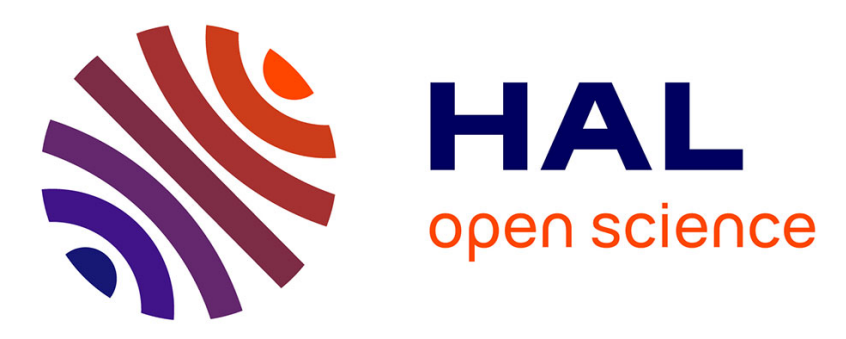

\title{
Stabilité des systèmes non linéaires sous échantillonnage apériodique
}

\author{
Hassan Omran, Laurentiu Hetel, Jean-Pierre Richard, Françoise
}

Lamnabhi-Lagarrigue

\section{- To cite this version:}

Hassan Omran, Laurentiu Hetel, Jean-Pierre Richard, Françoise Lamnabhi-Lagarrigue. Stabilité des systèmes non linéaires sous échantillonnage apériodique. Journal Européen des Systèmes Automatisés (JESA), 2013, 47 (4-8), pp.467-482. hal-01810883

\section{HAL Id: hal-01810883 \\ https://hal.science/hal-01810883}

Submitted on 6 Jul 2018

HAL is a multi-disciplinary open access archive for the deposit and dissemination of scientific research documents, whether they are published or not. The documents may come from teaching and research institutions in France or abroad, or from public or private research centers.
L'archive ouverte pluridisciplinaire HAL, est destinée au dépôt et à la diffusion de documents scientifiques de niveau recherche, publiés ou non, émanant des établissements d'enseignement et de recherche français ou étrangers, des laboratoires publics ou privés. 


\title{
Stabilité des systèmes non linéaires sous échantillonnage apériodique
}

\author{
Hassan Omran ${ }^{1}$, Laurentiu Hetel ${ }^{1}$, Jean-Pierre Richard ${ }^{1,2}$, \\ Françoise Lamnabhi-Lagarrigue ${ }^{3}$
}

\author{
1. LAGIS (CNRS UMR 8219), École Centrale de Lille, 59651 Villeneuve d'Ascq, \\ France \\ hassan.omran@ec-lille.fr,laurentiu.hetel@ec-lille.fr \\ 2. INRIA, Projet NON-A, Lille, France \\ jean-pierre.richard@ec-lille.fr \\ 3. Laboratoire des Signaux et Systèmes, European Embedded Control Institute \\ (EECI), SUPELEC, 3 rue Joliot Curie, 91192 Gif-sur-Yvette, France \\ lamnabhi@lss.supelec.fr
}

RÉSUMÉ. Cet article est dédié à l'analyse de la stabilité de systèmes non linéaires commandés par un contrôleur échantillonné non nécessairement périodique. Nous supposons qu'il existe une commande stabilisante en temps continu. Lors de l'implantation numérique de cette commande, il s'agit de trouver des conditions préservant la stabilité asymptotiquelexponentielle sous échantillonnage. Les conditions sont formulées à la fois pour la stabilité globale et la stabilité locale. Nous estimons une borne supérieure des pas d'échantillonnage dans le cas asynchrone (non périodique). L'idée principale est d'aborder le problème dans le cadre de la dissipativité. Le résultat est ensuite repris dans le cas spécifique des systèmes non linéaires polynomiaux, où les conditions de stabilité sont vérifiées numériquement en utilisant la décomposition en somme des carrés (SOS) et la programmation semi-définie.

ABSTRACT. This paper concerns the stability analysis of nonlinear sampled-data systems. Assuming the existence of a stabilizing continuous-time controller, we intend to provide sufficient asymptotic/exponential stability conditions for the digital implementation with asynchronous sampled-data. An allowable upper bound of the sampling interval is provided. The stability analysis problem is formulated both globally and locally. The main idea is to address the problem in the framework of dissipativity theory. Furthermore, the result is particularized for the class of polynomial sampled-data systems, where stability may be tested numerically using sum of squares decomposition and semidefinite programming.

MOTS-CLÉS : contrôleur échantillonné, stabilité, système non linéaire, dissipativité, Lyapunov. KEYWORDS: sampled-data control, stability, nonlinear systems, dissipativity, Lyapunov. 


\section{Introduction}

La stabilité des systèmes non linéaires échantillonnés est un problème difficile mais intéressant, puisque la mise en œuvre pratique des contrôleurs est généralent numérique. L'approche de l'émulation est souvent considérée. Dans cette approche, une commande qui stabilise le système en temps continu est implémentée en utilisant un bloqueur d'ordre zéro (BOZ). Intuitivement, on se doute que des pas d'échantillonnage "suffisamment petits" vont assurer la stabilité : au-delà de cette approche qualitative, il est concrètement important d'avoir une estimation quantitative de ce qu'on appelle le plus grand pas d'échantillonnage permis. Plusieurs travaux de la littérature se sont penchés sur ce problème (voir par exemple (Nešić et al., 2009), (Karafyllis, Kravaris, 2008) et (Karafyllis, Kravaris, 2009)). Le cas des systèmes échantillonnés linéaires a été largement étudié : en utilisant des fonctionnelles de LyapunovKrasovskii et une approche par retard d'entrée (Fridman et al., 2004), (Fridman, 2010), (Seuret, 2012); ou bien des techniques issues de la commande robuste (Mirkin, 2007), (Fujioka, 2009); ou encore une approximation polytopique et fonctions de Lyapunov-Razumikhin (Hetel et al., 2011), (Fiter et al., 2012).

Quelques travaux plus rares traitent le cas des systèmes nonlinéaires. Dans (Nešić et al., 2009), les résultats génériques sur les systèmes commandés en réseau (NCS) sont spécialisés pour le cas de systèmes échantillonnés : des résultats locaux et globaux sont présentés, ils sont basés sur des systèmes hybrides. Dans (Bauer et al., 2012), la stabilité asymptotique des NCS est étudiée en utilisant la même formulation de systèmes hybrides; les fonctions de Lyapunov sont construites par des techniques de somme des carrés (SOS en abréviation de l'anglais). Dans (Karafyllis, Kravaris, 2009), la notion d'accessibilité séquentielle permet de construire une commande garantissant la stabilisation globale robuste, et l'approche par retard d'entrée est exploitée pour le cas non linéaire.

La notion de dissipativité a été introduite par Willems (Willems, 1972). Depuis son introduction, cette approche a attiré beaucoup d'attention, car elle peut être utilisée pour étudier la stabilité, la passivité, la robustesse et d'autres problèmes d'analyse et de synthèse. Ces travaux sont inspirés par les propriétés de passivité des circuits électriques, et peuvent être vus comme la généralisation d'une notion abstraite d'énergie pour les systèmes dynamiques.

Récemment, dans (Omran et al., 2012), nous avons étudié la stabilité asymptotique locale de systèmes échantillonnés bilinéaires commandés par retour d'état linéaire statique, en utilisant l'analyse des sous-ensembles invariants et le théorème de la dissipativité. Les résultats sont prometteurs, mais ne sont pas trivialement applicables pour les systèmes non linéaires.

L'objectif de ce travail est d'étendre nos résultats (Omran et al., 2012) au cas non linéaire. Des conditions suffisantes, basées sur la dissipativité, sont présentées à la fois pour la stabilité asymptotique ou exponentielle, locale ou globale. La robustesse par rapport à la variation du pas d'échantillonnage est également considérée. De plus, nous étudierons le cas particulier des systèmes polynomiaux, où des techniques SOS 


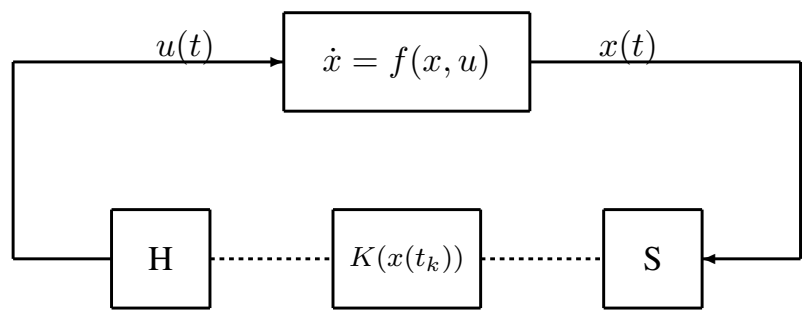

FIGURE 1. Commande du système non linéaire avec retour d'état échantillonné

(somme des carrés) seront utilisées pour extraire des fonctions de stockage et d'alimentation.

L'article est organisé comme suit : le problème est formalisé dans la section 2; dans la section 3, le système est représenté par un modèle équivalent permettant l'analyse de dissipativité ; la section 4 présente des conditions suffisantes de stabilité asymptotique/exponentielle; enfin, la section 5 illustre les résultats proposés sur deux exemples de systèmes non linéaires.

Définitions et notations : $\mathbb{R}^{n}$ désigne l'espace euclidien de dimension $n, \mathbb{R}^{n \times m}$ l'ensemble des matrices réelles de dimension $n \times m, P^{T}$ la transposée de $P \in \mathbb{R}^{n \times m}$, $\|x\|$ la norme $L_{2}$ d'un signal $x(\cdot) .\|\Delta\|$ désigne la norme $L_{2}$-induite de l'opérateur $\Delta$. Pour $P \in \mathbb{R}^{n \times n}, P>0$ indique que $P$ est définie positive, $P \geq 0$ indique qu'elle semi-définie positive. La notation $p(x) \in \mathbb{R}[x]$, avec $x \in \mathbb{R}^{n}$, indique que $p(x)$ appartient à l'ensemble des polynômes à coefficients dans $\mathbb{R}$ et à une indéterminée $\left\{x_{1}, x_{2}, \cdots, x_{n}\right\}$. Pour $x_{1}$ et $x_{2} \in \mathbb{R}^{n}$, on note $\left(x_{1}, x_{2}\right)=\left[x_{1}^{T}, x_{2}^{T}\right]^{T}$. Une fonction $\beta: \mathbb{R}_{\geq 0} \rightarrow \mathbb{R}_{\geq 0}$ est dite de classe $\mathcal{K}$ si elle est continue, nulle en zéro et strictement croissante ; de classe $\mathcal{K}_{\infty}$ si elle est de classe $\mathcal{K}$ et non bornée. $\beta: \mathbb{R}_{\geq 0} \times \mathbb{R}_{\geq 0} \rightarrow \mathbb{R}_{\geq 0}$ est de classe $\mathcal{K} L$ si $\forall t \geq 0, \beta(\cdot, t)$ est de classe $\mathcal{K}$ et si $\forall s \geq 0, \beta(s, \cdot)$ est noncroissante avec $\lim _{t \rightarrow \infty} \beta(s, t)=0$.

\section{Formulation du problème}

On considère le système non linéaire :

$$
\dot{x}(t)=f(x(t), u(t)), \quad \forall t>t_{0}, \quad x\left(t_{0}\right)=x_{0},
$$

où $x(t) \in \mathbb{R}^{n}$ et $u(t) \in \mathbb{R}^{m}$ sont respectivement l'état et l'entrée. La fonction $f$ : $\mathbb{R}^{n} \rightarrow \mathbb{R}^{n}$, avec $f(0,0)=0$, est suffisamment lisse pour qu'à chaque $x\left(t_{0}\right)$ et $u(\cdot)$ admissible corresponde une et une seule solution sur $\left[t_{0}, \infty\right)$.

On suppose qu'il existe une commande $u(t)=K(x(t))$ qui stabilise l'équilibre $x=0$ en temps continu, où $K: \mathbb{R}^{n} \rightarrow \mathbb{R}^{m}$ est une fonction continûment différentiable. On considère les définitions de stabilité suivantes. 
DÉFINITION 1. - Le point d'équilibre $x=0$ de (1) est localement uniformément asymptotiquement stable dans un voisinage $\mathcal{L}$ de l'équilibre, s'il existe une fonction $\beta(\cdot, \cdot)$ de classe $\mathcal{K} L$, telle que

$$
|x(t)| \leq \beta\left(\left|x\left(t_{0}\right)\right|, t-t_{0}\right), \quad \forall t \geq t_{0}, \quad \forall x\left(t_{0}\right) \in \mathcal{L} .
$$

Il est globalement uniformément asymptotiquement stable si (2) est satisfaite pour $\mathcal{L}=\mathbb{R}^{n}$.

DÉFINITION 2. - Le point d'équilibre $x=0$ de (1) est localement exponentiellement stable dans un voisinage $\mathcal{L}$ de l'équilibre, si (2) est satisfaite avec:

$$
\beta(s, t)=c s e^{-\lambda t}, \quad c>0, \lambda>0,
$$

et globalement exponentiellement stable si de plus $\mathcal{L}=\mathbb{R}^{n}$.

On considère l'émulation de la commande $u=K(x)$ en supposant que :

- les instants d'échantillonnage $\left\{0=t_{0}<t_{1}, \ldots<t_{k}<\ldots\right\}$ tendent vers l'infini et les pas d'échantillonnage correspondants ont une borne supérieure finie $h_{\max }$ :

$$
0<t_{k+1}-t_{k} \leq h_{\max }, \quad \forall k \in \mathbb{N}, \quad \lim _{k \rightarrow \infty} t_{k}=\infty
$$

- le contrôle est un retour d'état constant par morceaux :

$$
u(t)=K\left(x\left(t_{k}\right)\right), \quad \forall t \in\left[t_{k}, t_{k+1}\right) .
$$

On obtient alors le système en boucle fermée (figure 1) :

$$
\dot{x}(t)=f\left(x(t), K\left(x\left(t_{k}\right)\right)\right), \quad \forall t \in\left[t_{k}, t_{k+1}\right), \quad k \in \mathbb{N} .
$$

Problème : Notre objectif est de trouver un critère de stabilité asymptotique et exponentielle locale/globale de l'équilibre $x=0$ du système non linéaire échantillonné (3).

\section{Une représentation équivalente}

On note que le système (3) s'écrit aussi :

$$
\left\{\begin{array}{l}
\dot{x}(t)=f(x(t), K(x(t))+w(t)), \quad \forall t \in\left[t_{k}, t_{k+1}\right), \\
w(t)=K\left(x\left(t_{k}\right)\right)-K(x(t)),
\end{array}\right.
$$

ce qui montre que le système échantillonné (3) peut être représenté par le bouclage du système :

$$
\left\{\begin{array}{l}
\dot{x}=f(x, K(x)+w) \\
y=\frac{\partial K}{\partial x} \dot{x}
\end{array}\right.
$$




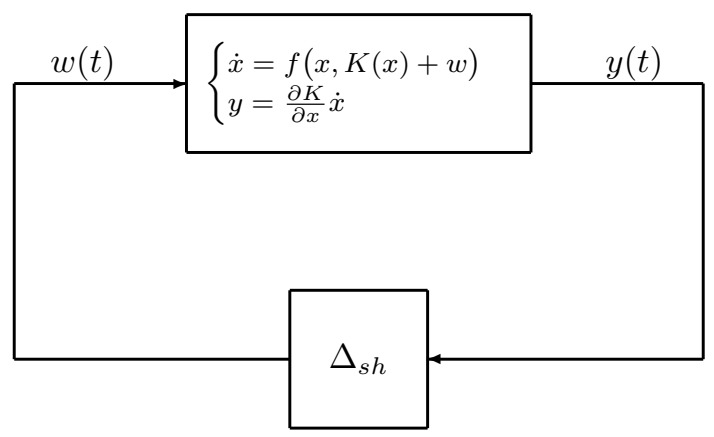

FIGURE 2. Représentation équivalente du système échantillonné (3)

avec l'opérateur $\Delta_{s h}: y \rightarrow w$ défini par :

$$
w(t)=\left(\Delta_{s h} y\right)(t)=-\int_{t_{k}}^{t} y(\tau) d \tau, \quad \forall t \in\left[t_{k}, t_{k+1}\right) \quad .
$$

Cette représentation est montrée dans la figure 2. On remarque que l'effet des variations des pas d'échantillonnage est modélisé par l'opérateur $\Delta_{s h}$. Cette approche est considérée dans (Mirkin, 2007) et (Fujioka, 2009) avec l'objectif d'étudier la stabilité des systèmes échantillonnés linéaires. Dans (Mirkin, 2007), une limite supérieure sur le gain de l'opérateur $\Delta_{s h}$ est trouvée. Il est montré que $\left\|\Delta_{s h}\right\| \leq \delta_{0}$ avec $\delta_{0}=\frac{2}{\pi} h_{\max }$. Cette limite est atteinte $\left(\left\|\Delta_{s h}\right\|=\delta_{0}\right)$ pour $t_{k+1}-t_{k}=h_{\max }$. Des conditions de stabilité basées sur le théorème du petit gain sont fournies sous la forme d'inégalités matricielles linéaires (LMI). Dans (Fujioka, 2009), la propriété précédente est associée à une propriété de passivité pour trouver des conditions moins contraignantes. Le résultat est basé sur des techniques de commande robuste, utilisant une approche fréquentielle et le lemme de Kalman-Yakubovich-Popov.

Les méthodes de (Mirkin, 2007) et (Fujioka, 2009) sont développées pour les systèmes linéaires invariants dans le temps (LTI), mais ne sont pas applicables pour le cas non linéaire. Dans (Omran et al., 2012), les propriétés de l'opérateur sont utilisées pour étudier la stabilité de systèmes bilinéaires échantillonnés, en utilisant le théorème de dissipativité. Les lemmes suivants y sont démontrés, se basant sur le travail (Fujioka, 2009).

Lemme 3. - (Omran et al., 2012) Soit $\Delta_{\text {sh }}$ l'opérateur défini en (6). Pour tous y $\in$ $L_{2}\left[0, h_{\max }\right)$ et $0<X^{T}=X \in \mathbb{R}^{m \times m}$, l'inégalité suivante est satisfaite pour $t \in\left[t_{k}, t_{k+1}\right):$

$$
\boldsymbol{I}_{1}(t)=\int_{t_{k}}^{t}\left(\Delta_{s h} y\right)^{T}(\tau) X\left(\Delta_{s h} y\right)(\tau)-\delta_{0}^{2} y^{T}(\tau) X y(\tau) d \tau \leq 0 .
$$


PREUVE 4. - D'abord on remarque que puisque $X^{T}=X>0$ alors il existe $U \in$ $\mathbb{R}^{n \times n}$ telle que $X=U^{T} U$. Pour $t \in\left[t_{k}, t_{k+1}\right)$ on a

$$
\boldsymbol{I}_{1}(t)=\int_{t_{k}}^{t}\left(U\left(\Delta_{s h} v\right)(\tau)\right)^{T}\left(U\left(\Delta_{s h} v\right)(\tau)\right)-\delta_{0}^{2}(U v(\tau))^{T}(U v(\tau)) d \tau .
$$

Par (6) on remarque que $U\left(\Delta_{s h} v\right)=\Delta_{s h}(U v)$, alors

$$
\boldsymbol{I}_{1}(t)=\int_{t_{k}}^{t}\left(\left(\Delta_{s h}(U v)\right)(\tau)\right)^{T}\left(\left(\Delta_{s h}(U v)\right)(\tau)\right)-\delta_{0}^{2}(U v(\tau))^{T}(U v(\tau)) d \tau .
$$

En définissant le vecteur $z=U v \in L_{2}\left[0, h_{\max }\right)$, on obtient

$$
\boldsymbol{I}_{1}(t)=\int_{t_{k}}^{t}\left(\Delta_{s h} z\right)^{T}(\tau)\left(\Delta_{s h} z\right)(\tau) d \tau-\delta_{0}^{2} \int_{t_{k}}^{t} z^{T}(\tau) z(\tau) d \tau
$$

Le dernier terme est négatif car $\left\|\Delta_{s h}\right\| \leq \delta_{0}$.

LEMME 5. - (Omran et al., 2012) Soit $\Delta_{\text {sh }}$ l'opérateur défini en (6). Pour tous y $\in$ $L_{2}\left[0, h_{\max }\right)$ et $0 \leq Y^{T}=Y \in \mathbb{R}^{m \times m}$, l'inégalité suivante est satisfaite pour $t \in$ $\left[t_{k}, t_{k+1}\right)$ :

$$
\boldsymbol{I}_{2}(t)=\int_{t_{k}}^{t}\left(\Delta_{s h} y\right)(\tau)^{T} Y y(\tau)+y^{T}(\tau) Y\left(\Delta_{s h} y\right)(\tau) d \tau \leq 0 .
$$

Preuve 6. - Pour $t \in\left(t_{k}, t_{k+1}\right)$ on a $\frac{d}{d t}\left(\Delta_{s h} v\right)(t)=-v(t)$, alors

$$
\begin{aligned}
\boldsymbol{I}_{2}(t) & =2 \int_{t_{k}}^{t} v^{T}(\tau) Y\left(\Delta_{s h} v\right)(\tau) d \tau \\
& =-\int_{t_{k}}^{t} \frac{d}{d \tau}\left(\left(\Delta_{s h} v\right)^{T}(\tau) Y\left(\Delta_{s h} v\right)(\tau)\right) d \tau \\
& =\left[-\left(\Delta_{s h} v\right)^{T}(\tau) Y\left(\Delta_{s h} v\right)(\tau)\right]_{t_{k}}^{t}=-\left(\Delta_{s h} v\right)^{T}(t) Y\left(\Delta_{s h} v\right)(t) \leq 0 .
\end{aligned}
$$

Nous proposons ici d'exploiter ces lemmes afin de développer un critère de stabilité pour le contrôle échantillonné des systèmes non linéaires (non limités aux bilinéaires). Notre approche s'inspire de la notion de dissipativité exponentielle définie dans (Chellaboina, Haddad, 2003).

\section{Principaux résultats}

\subsection{Analyse de stabilité}

THÉORÈME 7. - Soient le système non linéaire échantillonné (3) et sa représentation équivalente (5), (6). On définit $\delta_{0}=\frac{2}{\pi} h_{\max }$ et on introduit la forme quadratique : 


$$
\boldsymbol{S}(y(t), w(t))=\left[\begin{array}{c}
y(t) \\
w(t)
\end{array}\right]^{T}\left[\begin{array}{rr}
-\delta_{0}^{2} X & Y \\
Y & X
\end{array}\right]\left[\begin{array}{c}
y(t) \\
w(t)
\end{array}\right],
$$

avec $0<X^{T}=X \in \mathbb{R}^{m \times m}$ et $0 \leq Y^{T}=Y \in \mathbb{R}^{m \times m}$. Considérons un voisinage $\mathcal{D} \subset \mathbb{R}^{n}$ de l'équilibre $x=0$ et une fonction différentiable définie positive $V: \mathcal{D} \rightarrow$ $\mathbb{R}^{+}$pour laquelle il existe des fonctions $\beta_{1}$ et $\beta_{2}$ de classe $\mathcal{K}$, telles que :

$$
\beta_{1}(|x|) \leq V(x) \leq \beta_{2}(|x|), \quad \forall x \in \mathcal{D} .
$$

Si, pour un $\alpha>0$ et pour tout $x(t) \in \mathcal{D}, V$ satisfait :

$$
\begin{aligned}
\dot{V}(x(t))+\alpha V(x(t)) & \leq \boldsymbol{S}(y(t), w(t)), \\
\dot{V}(x(t))+\alpha V(x(t)) & \leq \boldsymbol{S}(y(t), w(t)) e^{-\alpha h_{\max }},
\end{aligned}
$$

alors l'équilibre $x=0$ du système (3) est localement uniformément asymptotiquement stable.

De plus, considérons les ensembles définis par $V(\cdot)$ et un scalaire $c>0$ :

$$
\mathcal{L}_{c}:=\left\{x \in \mathbb{R}^{n}: V(x) \leq c\right\} .
$$

Alors, l'ensemble $\mathcal{L}_{c^{*}}$ défini par la surface de niveau maximal de $V$ contenue dans $\mathcal{D}$ :

$$
c^{*}=\max _{\mathcal{L}_{c} \subset \mathcal{D}} c
$$

est une estimation de domaine d'attraction de $x=0$.

Enfin, si toutes les conditions sont satisfaites globalement avec des fonctions $\beta_{1}$ et $\beta_{2}$ de classe $\mathcal{K}_{\infty}$, alors $x=0$ est globalement uniformément asymptotiquement stable.

PReUve 8. - Pour montrer la stabilité du système échantillonné, on considère d'abord la fonction :

$$
W(t)=V(x(t)) e^{\alpha\left(t-t_{k}\right)}-\int_{t_{k}}^{t} \boldsymbol{S}(y(\tau), w(\tau)),
$$

pour $t \in\left[t_{k}, t_{k+1}\right)$. Sa dérivée temporelle est :

$$
\begin{aligned}
\dot{W}(t) & =[\dot{V}(x(t))+\alpha V(x(t))] e^{\alpha\left(t-t_{k}\right)}-\boldsymbol{S}(y(t), w(t)), \\
& =\left[\dot{V}(x(t))+\alpha V(x(t))-\boldsymbol{S}(y(t), w(t)) e^{-\alpha\left(t-t_{k}\right)}\right] e^{\alpha\left(t-t_{k}\right)} .
\end{aligned}
$$

Remarquons que pour tout $t \in\left[t_{k}, t_{k+1}\right)$, le terme $e^{-\alpha\left(t-t_{k}\right)}$ est dans $\left[e^{-\alpha\left(h_{\max }\right)}, 1\right]$ et il existe $\theta(t) \in[0,1]$ tel que $e^{-\alpha\left(t-t_{k}\right)}=\theta(t)+(1-\theta(t)) e^{-\alpha h_{\max }}$. 
En multipliant (11) et (12) respectivement par $\theta(t)$ et $1-\theta(t)$ et en prenant la somme des inégalités obtenues, on obtient :

$$
\begin{aligned}
\dot{V}(x(t))+\alpha V(x(t))-\boldsymbol{S}(y(t), w(t)) e^{-\alpha\left(t-t_{k}\right)} & \leq 0, \\
\forall t \in\left[t_{k}, t_{k+1}\right), \quad \forall x(t) & \in \mathcal{D} .
\end{aligned}
$$

À partir de (15) et (16) on obtient :

$$
\dot{W}(t) \leq 0, \quad \forall t \in\left[t_{k}, t_{k+1}\right), \quad \forall x(t) \in \mathcal{D} .
$$

En intégrant $\dot{W}(\cdot)$ de $t_{k}$ à $t$ il vient :

$$
\begin{aligned}
& \int_{t_{k}}^{t} \dot{W}(s) d s \leq 0 \Rightarrow W(t) \leq W\left(t_{k}\right), \\
& V(x(t)) e^{\alpha\left(t-t_{k}\right)}-\int_{t_{k}}^{t} \boldsymbol{S}(y(\tau), w(\tau)) \leq V\left(t_{k}\right) .
\end{aligned}
$$

Par les Lemmes 3 et 5, on peut voir que le terme intégral dans la dernière équation satisfait :

$$
\int_{t_{k}}^{t} \boldsymbol{S}(y(\tau), w(\tau))=\boldsymbol{I}_{1}(t)+\boldsymbol{I}_{2}(t) \leq 0, \forall t \in\left[t_{k}, t_{k+1}\right),
$$

où $\boldsymbol{I}_{1}(t)$ et $\boldsymbol{I}_{2}(t)$ sont définis respectivement dans (7) et (8). À partir de (19) et (18) on obtient :

$$
V(x(t)) \leq e^{-\alpha\left(t-t_{k}\right)} V\left(x\left(t_{k}\right)\right), \quad \forall t \in\left[t_{k}, t_{k+1}\right), \forall x(t) \in \mathcal{D} .
$$

L'ensemble $\mathcal{L}_{c^{*}}$ est clairement positivement invariant (Khalil, 2002), il est le plus grand ensemble de niveau contenu dans $\mathcal{D}$. Considérons une condition initiale $x_{0} \in$ $\mathcal{L}_{c^{*}}$. En prolongeant, (20) entraîne :

$$
V(x(t)) \leq e^{-\alpha\left(t-t_{0}\right)} V\left(x\left(t_{0}\right)\right), \quad \forall t \geq t_{0}, \quad \forall x_{0} \in \mathcal{L}_{c^{*}} .
$$

De (10) et (21), on déduit que toute solution issue de $x\left(t_{0}\right) \in \mathcal{L}_{c^{*}}$ satisfait :

$$
\begin{aligned}
|x(t)| & \leq \beta_{1}^{-1}\left(V\left(x\left(t_{0}\right)\right) e^{-\alpha\left(t-t_{0}\right)}\right) \\
& \leq \beta_{1}^{-1}\left(\beta_{2}\left(\left|x\left(t_{0}\right)\right|\right) e^{-\alpha\left(t-t_{0}\right)}\right) \\
& :=\beta\left(\left|x\left(t_{0}\right)\right|, t-t_{0}\right), \quad \forall t \geq t_{0}, \forall x\left(t_{0}\right) \in \mathcal{L}_{c^{*}} .
\end{aligned}
$$

Il est facile de voir que la fonction $\beta(\cdot, \cdot)$ est de classe $\mathcal{K} L$. Alors, $x=0$ est localement uniformément asymptotiquement stable. Finalement, si toutes les conditions sont satisfaites globalement, avec des fonctions $\beta_{1}$ et $\beta_{2}$ de classe $\mathcal{K}_{\infty}$, alors $x=0$ est globalement uniformément asymptotiquement stable. 
Corollaire 9. - Supposons que toutes les conditions du Théorème 7 sont satisfaites pour :

$$
\beta_{1}(|x|) \geq k_{1}|x|^{q}, \beta_{2}(|x|) \leq k_{2}|x|^{q} \text {, avec } k_{1}, k_{2}, q>0 .
$$

Alors, l'équilibre $x=0$ est localement exponentiellement stable. De plus, l'ensemble $\mathcal{L}_{c^{*}}$ défini dans (14) et (13), est une estimation de domaine d'attraction. Si les conditions sont satisfaites globalement, alors $x=0$ est globalement exponentiellement stable.

PREUVE 10. - En suivant les mêmes étapes que dans la preuve du Théorème 7, on obtient :

$$
V(x(t)) \leq e^{-\alpha\left(t-t_{0}\right)} V\left(x\left(t_{0}\right)\right), \quad \forall t \geq t_{0}, \quad \forall x_{0} \in \mathcal{L}_{c^{*}},
$$

puis, par (10) et (22) :

$$
\begin{aligned}
|x(t)| & \leq\left(\frac{V\left(x\left(t_{0}\right)\right) e^{-\alpha\left(t-t_{0}\right)}}{k_{1}}\right)^{1 / q} \leq\left(\frac{k_{2}\left|x\left(t_{0}\right)\right|^{q} e^{-\alpha\left(t-t_{0}\right)}}{k_{1}}\right)^{1 / q} \\
& =\left(\frac{k_{2}}{k_{1}}\right)^{1 / q}\left|x\left(t_{0}\right)\right| e^{-(\alpha / q)\left(t-t_{0}\right)}, \quad \forall t \geq t_{0}, \quad \forall x\left(t_{0}\right) \in \mathcal{L}_{c^{*}} .
\end{aligned}
$$

Ainsi, $x=0$ est localement exponentiellement stable. Si toutes les conditions sont satisfaites globalement, la stabilité exponentielle globale est directe.

dissipatif (Chellaboina, Haddad, 2003) à la fois par rapport aux fonctions d'alimentation $\boldsymbol{S}(y, w)$ de (9), et $e^{-\alpha h_{\max }} \boldsymbol{S}(y, w)$.

\subsection{Cas particulier des systèmes polynomiaux}

Plusieurs phénomènes physiques (contrôle de procédés, biologie, robotique, systèmes électriques) peuvent être modélisés ou approchés par des équations différentielles polynomiales. Pour cette classe, la décomposition en somme des carrés et la programmation semi-définie (Parrilo, 2000) sont des techniques très utiles, déjà utilisées dans plusieurs problèmes d'analyse et de synthèse (Papachristodoulou, Prajna, 2005).

Dans cette section, nous spécialiserons les résultats précédents au cas des systèmes échantillonnés polynomiaux. Cette restriction nous permettra de proposer une méthode constructive pour la recherche de fonctions de stockage et d'alimentation satisfaisant les conditions de stabilité asymptotique/exponentielle proposées dans la section précédente.

Considérons le problème de stabilité défini dans la section 2 pour le cas particulier où $f(x, u)$ et $K(x)$ sont des fonctions polynomiales. Le système (5) est défini par des fonctions polynomiales $F(x, w):=f(x, K(x)+w)$ et $G(x, w):=\frac{\partial K}{\partial x} F(x, w)$ :

$$
\left\{\begin{array}{l}
\dot{x}=F(x, w), \\
y=G(x, w) .
\end{array}\right.
$$


Vérifier les inégalités de dissipativité du Théorème 7 revient à un problème de test de négativité sur des polynômes. Ceci se constate sur les équations (9) et (23), puisque (11) et (12) sont respectivement équivalentes à :

$$
\begin{aligned}
0 \leq & -\frac{\partial V}{\partial x} F(x, w)-\alpha V(x) \\
& +\left[-\delta_{0}^{2} G^{T}(x, w) X G(x, w)+2 G^{T}(x, w) Y w+w^{T} Y w\right],
\end{aligned}
$$

et

$$
\begin{aligned}
0 \leq & -\frac{\partial V}{\partial x} F(x, w)-\alpha V(x) \\
& +\left[-\delta_{0}^{2} G^{T}(x, w) X G(x, w)+2 G^{T}(x, w) Y w+w^{T} Y w\right] e^{-\alpha h_{\max }}
\end{aligned}
$$

pour tout $x(t) \in \mathcal{D}$. En fait, les dernières inégalités concernent des polynômes de la forme $p(\xi) \geq 0$, où $p(\xi) \in \mathbb{R}[\xi]$, et $\xi=(x, w)$.

Il est bien connu que la vérification de positivité d'un polynôme multivarié est un problème difficile. Des méthodes récentes permettent cependant de simplifier ce problème en utilisant la décomposition en somme des carrés (SOS) et la programmation semi-définie. Ces méthodes testent si le polynôme est une somme des carrés, ce qui est suffisant pour assurer sa positivité semi-définie.

DÉFINITION 11. - (Papachristodoulou, Prajna, 2005) Un polynôme multivarié $p(x) \in \mathbb{R}[x]$ est une somme de carrés (SOS) s'il existe $p_{i}(x) \in \mathbb{R}[x], i \in\{1, \ldots, M\}$, tels que $p(x)=\sum_{i=1}^{M} p_{i}^{2}(x)$.

La littérature (voir l'article de synthèse (Papachristodoulou, Prajna, 2005)) semble suggérer que cette approche (conditions suffisantes) est finalement peu conservative. Néanmoins, on doit noter que la complexité des calculs pour tester si un polynôme $p(x)$ est SOS augmente rapidement avec le degré de $p(x)$.

Dans ce qui suit, on reformule le Théorème 7 et le Corollaire 9 en utilisant une méthode de somme des carrés. L'applicabilité locale des conditions de dissipativité, dans une région $\mathcal{D}$, est garantie en utilisant une technique similaire à la $\mathrm{S}$-procédure (Boyd et al., 1994). Notons que l'on doit s'assurer que la fonction de stockage est définie positive et qu'il n'est donc pas suffisant de s'assurer qu'elle se compose en somme des carrés (qui ne garantit que la positivité semi-définie). Afin de régler ce problème, nous utiliserons la proposition suivante.

Proposition 12. - (Papachristodoulou, Prajna, 2005) Soient deux polynômes de degré $2 d, V(x) \in \mathbb{R}[x]$ et :

$$
\varphi(x)=\sum_{i=1}^{n} \sum_{j=1}^{d} \epsilon_{i j} x_{i}^{2 j}, \text { tel que } \sum_{j=1}^{d} \epsilon_{i j}>\gamma, \forall i=1, \ldots, n
$$

avec $\gamma>0$ et $\epsilon_{i j} \geq 0$ pour tout $i, j$. Alors, la condition :

$$
[V(x)-\varphi(x)] \text { est } S O S
$$


garantit que $V(x)$ est définie positive.

COROllaIRE 13. - On considère le système échantillonné (3) dans le cas où $f(x, u)$ et $K(x)$ sont des fonctions polynomiales, ainsi que la représentation (6), (23) et $\delta_{0}=\frac{2}{\pi} h_{\text {max }}$. Soit $\mathcal{D}=\left\{x \in \mathbb{R}^{n}: \mu_{l}(x) \geq 0, l=1,2, \ldots, s\right\}$ un voisinage de $x=0$. Supposons qu'il existe un polynôme $V(x) \in \mathbb{R}[x]$ et des sommes des carrés $\sigma_{l}(\xi)$ et $\varsigma_{l}(\xi)$, avec $l \in\{1, \ldots, s\}$ et $\xi=(x, w)$, tels que les polynômes suivants sont des sommes des carrés :

$$
\begin{aligned}
\hat{V}(x)= & V(x)-\varphi(x), \\
\rho_{1}(\xi)= & -\sum_{l=1}^{s} \sigma_{l}(\xi) \mu_{l}(x)-\frac{\partial V}{\partial x} F(x, w)-\alpha V(x) \\
& +\left[-\delta_{0}^{2} G^{T}(x, w) X G(x, w)+2 G^{T}(x, w) Y w+w^{T} Y w\right], \\
\rho_{2}(\xi)= & -\sum_{l=1}^{s} \varsigma_{l}(\xi) \mu_{l}(x)-\frac{\partial V}{\partial x} F(x, w)-\alpha V(x) \\
& +\left[-\delta_{0}^{2} G^{T}(x, w) X G(x, w)+2 G^{T}(x, w) Y w+w^{T} Y w\right] e^{-\alpha h_{\max }} .
\end{aligned}
$$

avec $0<X^{T}=X \in \mathbb{R}^{m \times m}, 0 \leq Y^{T}=Y \in \mathbb{R}^{m \times m}$ et $\varphi(x)$ un polynôme défini positif de type (24). Alors, l'équilibre $x=0$ du système (3) est localement uniformément asymptotiquement stable. De plus, l'ensemble $\mathcal{L}_{c^{*}}$ défini dans (13), (14), est une estimation du domaine d'attraction. Finalement, si (27) et (28) sont des sommes des carrés avec $\mu_{l}(x)=0$, et pour tout $l \in\{1,2, \ldots, s\}$, alors l'équilibre est globalement uniformément asymptotiquement stable.

PREUVE 14. - D'abord, on note que par (26) et la Proposition 12, la fonction $V(x)$ est définie positive et radialement non bornée $(V(x) \rightarrow \infty$ quand $\|x\| \rightarrow \infty)$. Donc, par le Lemme 4.3 de (Khalil, 2002), il existe des fonctions $\beta_{1}$ et $\beta_{2}$ de classe $\mathcal{K}$, telles que:

$$
\beta_{1}(|x|) \leq V(x) \leq \beta_{2}(|x|), \quad \forall x \in \mathbb{R}^{n} .
$$

Quand $x(t) \in \mathcal{D}$, autrement dit $\mu_{l}(x) \geq 0$ pour tout $l \in\{1,2, \ldots, s\}$, par la positivité de $\sigma_{l}(\xi)\left(\varsigma_{l}(\xi)\right)$ on a $\rho_{1}(\xi) \geq 0\left(\rho_{2}(\xi) \geq 0\right)$. Par conséquent, toutes les conditions $d u$ Théorème 7 sont satisfaites. Le cas où (27) et (28) sont des sommes de carrés pour $\mu_{l}(x)=0 \forall l \in\{1,2, \ldots, s\}$ satisfait clairement les conditions de stabilité globale du Théorème 7 .

COROllaire 15. - Supposons que toutes les conditions du Corollaire 13 soient satisfaites et que la fonction de stockage $V(x)$ satisfasse :

$$
k_{1}|x|^{q} \leq V(x) \leq k_{2}|x|^{q}, \quad \forall x \in \mathbb{R}^{n} .
$$

Alors, l'équilibre $x=0$ est localement exponentiellement stable. De plus, l'ensemble $\mathcal{L}_{c^{*}}$ défini dans (13), (14) est une estimation du domaine d'attraction. Si les conditions sont satisfaites globalement, alors $x=0$ est globalement exponentiellement stable.

PREUVE 16. - La preuve suit les mêmes étapes que le Corollaire 13 et est un résultat direct du Corollaire 9. 


\section{Exemples}

On applique maintenant les méthodes proposées sur deux systèmes non linéaires. D'abord, on considère un exemple de (Nešić et al., 2009), où l'on cherche le plus grand pas d'échantillonnage préservant la stabilité globale asymptotique du système échantillonné. Ensuite, on considèrera un autre exemple qui illustrera l'applicabilité des résultats pour étudier la stabilité locale exponentielle.

\subsection{Exemple 1}

On considère le système suivant, issu de (Nešić et al., 2009) :

$$
\dot{x}=d x^{2}-x^{3}+u,
$$

avec un paramètre variable et borné $|d| \leq 1$ et une commande $u=K(x)=-2 x$. L'émulation discrète de cette loi de commande donne un système échantillonné représenté par l'opérateur $\Delta_{s h}$ de (6) et le système (5) :

$$
\left\{\begin{array}{l}
\dot{x}=d x^{2}-x^{3}-2 x+w, \\
y=-2\left(d x^{2}-x^{3}-2 x+w\right) .
\end{array}\right.
$$

On applique le Corollaire 13 afin de trouver une fonction de stockage de la forme $V(x)=a x^{2}+b x^{4}$, telle que (26), (27) et (28) soient des sommes des carrés. On choisit $\varphi(x)=10^{-3} x^{2}, \alpha=0,1$ et $h_{\max }=0,72$. Notre objectif est de vérifier la stabilité globale. Dans ce cas, les polynômes (27) et (28) sont :

$$
\begin{aligned}
\rho_{1}(\xi)= & -\left(2 a x+4 b x^{3}\right)\left(d x^{2}-x^{3}-2 x+w\right)-\alpha\left(a x^{2}+a x^{4}\right) \\
& +\left[-4 \delta_{0}^{2} X\left(d x^{2}-x^{3}-2 x+w\right)^{2}\right. \\
& \left.-4 Y\left(d x^{2}-x^{3}-2 x+w\right) w+Y w^{2}\right], \\
\rho_{2}(\xi)= & -\left(2 a x+4 b x^{3}\right)\left(d x^{2}-x^{3}-2 x+w\right)-\alpha\left(a x^{2}+a x^{4}\right) \\
& +\left[-4 \delta_{0}^{2} X\left(d x^{2}-x^{3}-2 x+w\right)^{2}\right. \\
& \left.-4 Y\left(d x^{2}-x^{3}-2 x+w\right) w+Y w^{2}\right] e^{-\alpha h_{\max }},
\end{aligned}
$$

où $a, b, X, Y$ sont des variables à définir. Remarquons que les termes $d$ et $d^{2}$ apparaissent dans les polynômes. Cependant, si (30) et (31) sont des sommes des carrés pour tout $\left(d, d^{2}\right) \in\{(1,0),(1,1),(-1,0),(-1,1)\}$, alors ils sont des sommes des carrés pour tout $d(t)$ variant dans le temps $|d| \leq 1$. On le vérifie en utilisant le logiciel SOSTOOLS (S. Prajna, Parrilo, 2002) pour une fonction de stockage $V(x)=$ $0,7740 x^{2}+0,1991 x^{4}$ et pour une fonction d'alimentation (9) définie par $X=0,4752$ et $Y=0,623010^{-3}$. En appliquant le Corollaire 13, on obtient la stabilité globale uniforme asymptotique de l'équilibre $x=0$ du système échantillonné. Notons qu'il est impossible de trouver une solution en cherchant une fonction de stockage quadratique. En choisissant $\alpha$ (taux de décroissance de la fonction de stockage) plus 


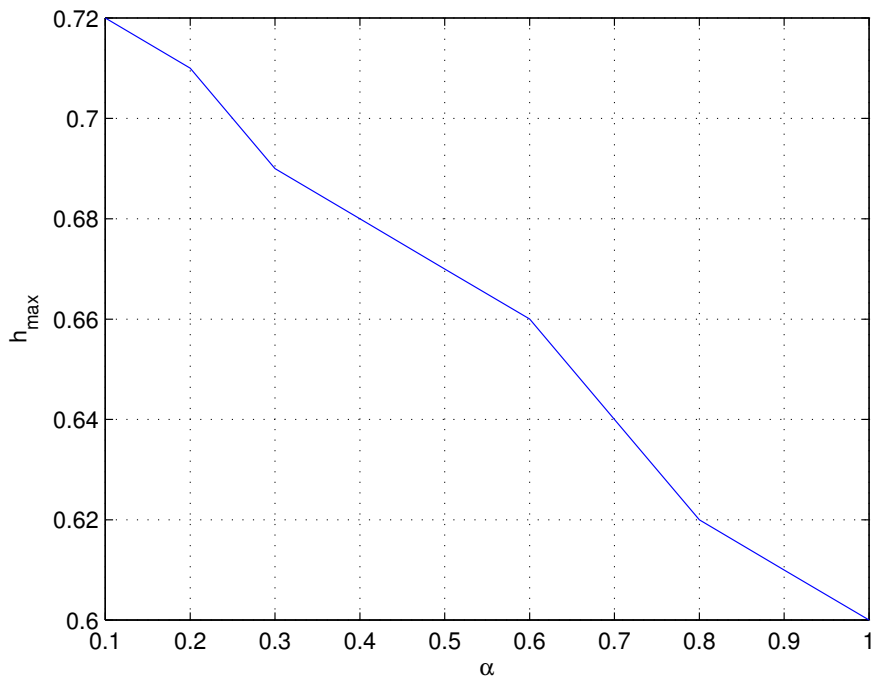

FIGURE 3. Compromis entre le taux de décroissance exponentielle $\alpha$ et l'intervalle d'échantillonage maximal $h_{\max }$

grand, on trouve que le problème a une solution pour un $h_{\max }$ plus petit (voir figure 3). Plusieurs travaux ont considéré cet exemple avec comme objectif d'estimer le plus grand pas d'échantillonnage conservant la stabilité. Les estimations obtenues sont $h_{\max }=0,368$ (Nešić et al., 2009) et $h_{\max }=0,1428$ (Karafyllis, Kravaris, 2008). Les conditions proposées dans ce travail sont satisfaites pour $h_{\max }=0,72$.

La simulation de l'évolution de l'état est montré dans figure 4. L'état converge asymptotiquement pour des intervalles d'échantillonnage variables inférieures à 0,72 . De plus, la stabilité asymptotique du système n'est plus garantie pour une intervalle d'échantillonnage constante $t_{k+1}-t_{k}=1,05$.

\subsection{Exemple 2}

On considère maintenant le système :

$$
\dot{x}=x^{2}+(x-1) u,
$$

avec la commande en temps continu $u=K(x)=x+2 x^{2}$ qui stabilise, seulement localement, l'équilibre $x=0$. Notre objectif est de trouver le plus grand $h_{\max }$ qui garantit la stabilité locale exponentielle de $x=0$ lorque la commande est émulée. On 

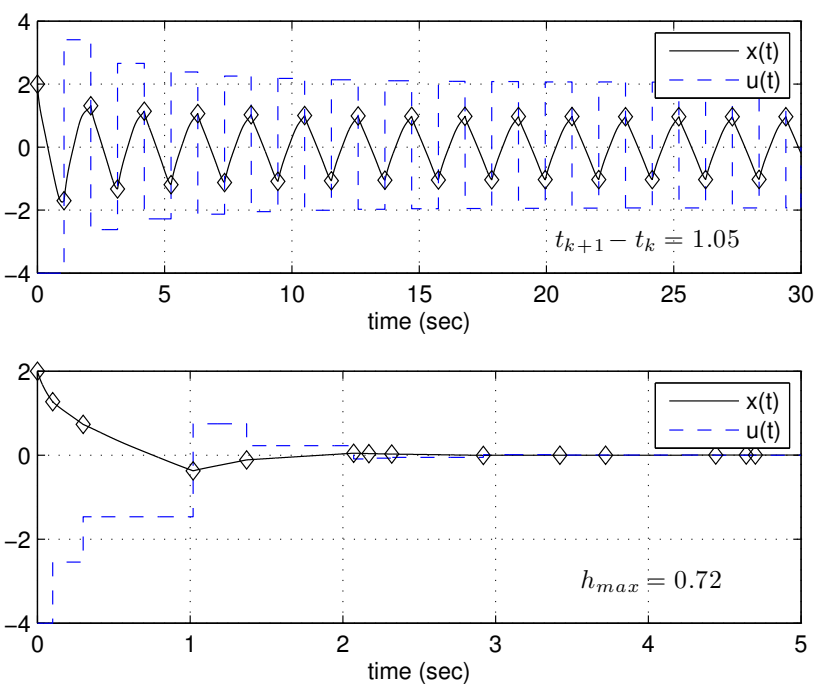

FIGURE 4. État du système pour deux sequence d'échantillonnage

considère le voisinage $x \in[-0,4,+0,4]$. Le système échantillonné peut être représenté par l'opérateur $\Delta_{s h}$ de (6) et le système (5) décrit par :

$$
\left\{\begin{array}{l}
\dot{x}=-x+2 x^{3}+(x-1) w \\
y=(1+4 x)\left(-x+2 x^{3}+(x-1) w\right) .
\end{array}\right.
$$

On applique le Corollaire 15 avec une fonction de stockage quadratique $V(x)=$ $a x^{2}$. Remarquons que $V(x)$ satisfait (29) avec $k_{1}=k_{2}=a$ et $q=2$. On choisit $\varphi(x)=10^{-3} x^{2}, \alpha=0,25$ et $h_{\text {max }}=0,6$. Le domaine considéré $\mathcal{D}$ est décrit par $\left\{x \in \mathbb{R}: \mu_{1}(x) \geq 0\right\}$ avec $\mu_{1}(x)=(x+0,4)(0,4-x)$. Dans ce cas, les polynômes (27) et (28) sont :

$$
\begin{aligned}
\rho_{1}(\xi)= & -\sigma_{1}(\xi) \mu_{1}(x)-(2 a x)\left(-x+2 x^{3}+(x-1) w\right)-\alpha\left(a x^{2}\right) \\
& +\left[-\delta_{0}^{2} X(1+4 x)^{2}\left(-x+2 x^{3}+(x-1) w\right)^{2}\right. \\
& \left.+2 Y(1+4 x)\left(-x+2 x^{3}+(x-1) w\right) w+Y w^{2}\right], \\
\rho_{2}(\xi)= & -\varsigma_{1}(\xi) \mu_{1}(x)-(2 a x)\left(-x+2 x^{3}+(x-1) w\right)-\alpha\left(a x^{2}\right) \\
& +\left[-\delta_{0}^{2} X(1+4 x)^{2}\left(-x+2 x^{3}+(x-1) w\right)^{2}\right. \\
& \left.+2 Y(1+4 x)\left(-x+2 x^{3}+(x-1) w\right) w+Y w^{2}\right] e^{-\alpha h_{\max }},
\end{aligned}
$$


où $a, X, Y$ sont des variables à définir et les sommes de carrés $\sigma_{1}(\xi), \varsigma_{1}(\xi)$ sont des polynômes à définir. En utilisant le logiciel SOSTOOLS, on trouve que (32) et (33) sont des sommes de carrés avec $a=0,1201, X=0,2551, Y=0,884610^{-2}$ et les polynômes :

$$
\begin{aligned}
\sigma_{1}(\xi)= & 0,6234 w^{2}-0,3616 x w^{2}+1,6714 x^{2} w^{2} \\
& -0,6762 x^{3} w+2,0314 x^{4} w+3,228 x^{6}, \\
\varsigma_{1}(\xi)= & 0,5202 w^{2}-0,3169 x w^{2}+1,4349 x^{2} w^{2} \\
& -0,5482 x^{3} w+1,6075 x^{4} w+2,8846 x^{6} .
\end{aligned}
$$

Alors, toutes les conditions du Corollaire 15 sont satisfaites et $x=0$ est localement exponentiellement stable. Il est facile de voir que l'estimation de domaine d'attraction $\mathcal{L}_{c^{*}}$ est le domaine étudié $[-0,4,+0,4]$.

\section{Conclusion}

Dans cet article, nous avons proposé des conditions suffisantes pour la stabilité des systèmes non linéaires échantillonnés. L'idée principale est d'utiliser le théorème de dissipativité pour estimer le plus grand pas d'échantillonnage qui garantit la stabilité asymptotique/exponentielle. Ces résultats ont ensuite été particularisés pour les systèmes polynomiaux, pour lesquels la technique peut devenir constructive grâce à une décomposition SOS (en somme de carrés) associée à la programmation semi-définie. Un première illustration a montré l'intérêt de cette approche sur un exemple de la littérature; le second exemple a concerné la stabilisation exponentielle locale sous échantillonnage.

\section{Remerciements}

Ce travail a été soutenu financièrement par le Septième Programme-Cadre de l'Union Européenne FP7/2007-2013 sous l'accord n. 257462 : HYCON2 Network of Excellence "Highly-Complex and Networked Control Systems", par le programme de coopération Interreg IVA 2 mers seas Zeeën 2007-2013 sous le projet “SYSIASS 6-20”, et par le ministère de l'éducation supérieure et de la recherche, le conseil de la région Nord-Pas de Calais et FEDER, au moyen du "Contrat de projets État Région (CPER) 2007-2013”.

\section{Bibliographie}

Bauer N., Maas P., Heemels W. (2012). Stability analysis of networked control systems: A sum of squares approach. Automatica, vol. 48, no 8, p. 1514 - 1524.

Boyd S., Ghaoui L. E., Feron E., Balakrishnan V. (1994). Linear matrix inequalities in system and control theory. SIAM. 
Chellaboina V., Haddad W. M. (2003). Exponentially dissipative nonlinear dynamical systems: a nonlinear extension of strict positive realness. Mathematical Problems in Engineering, vol. 1, p. 25-45

Fiter C., Hetel L., Perruquetti W., Richard J.-P. R. (2012). A state dependent sampling for linear state feedback. Automatica, vol. 48, no 8, p. 1860 - 1867.

Fridman E. (2010). A refined input delay approach to sampled-data control. Automatica, vol. $46, n^{\circ} 2$, p. 421 - 427.

Fridman E., Seuret A., Richard J.-P. (2004). Robust sampled-data stabilization of linear systems: an input delay approach. Automatica, vol. 40, p. 1441-1446.

Fujioka H. (2009). Stability analysis of systems with aperiodic sample-and-hold devices. $A u$ tomatica, vol. 45, p. 771-775.

Hetel L., Kruszewski A., Perruquetti W., Richard J. (2011). Discrete and intersample analysis of systems with aperiodic sampling. IEEE Transactions on Automatic Control, vol. 56, $\mathrm{n}^{\circ} 7$, p. $1696-1701$.

Karafyllis I., Kravaris C. (2008, sep.). Global stability results for sytems under sampled-data control. Int. J. Robust Nonlin. Control, vol. 19, p. 1105-1128.

Karafyllis I., Kravaris C. (2009). Robust global stabilizability by means of sampled-data control with positive sampling rate. International Journal of Control, vol. 82, no 4, p. 755-772.

Khalil H. K. (2002). Nonlinear systems. Prentice-Hall, Upper Saddle River.

Mirkin L. (2007). Some remarks on the use of time-varying delay to model sample-and-hold circuits. IEEE Transactions on Automatic Control, vol. 52, nº 6, p. 1109-1112.

Nešić D., Teel A., Carnevale D. (2009). Explicit computation of the sampling period in emulation of controllers for nonlinear sampled-data systems. IEEE Transactions on Automatic Control, vol. 54, no 3, p. $619-624$.

Omran H., Hetel L., Richard J.-P. (2012, jun.). Local stability of bilinear systems with asynchronous sampling. In The 4th ifac conference on analysis and design of hybrid systems.

Papachristodoulou A., Prajna S. (2005, jun.). A tutorial on sum of squares techniques for systems analysis. In American control conference, vol. 4, p. 2686 - 2700.

Parrilo P. A. (2000). Structured semidefinite programs and semialgebraic geometry methods in robustness and optimization. Thèse de doctorat non publiée, California Institute of Technology, Pasadena, CA.

Seuret A. (2012). A novel stability analysis of linear systems under asynchronous samplings. Automatica, vol. 48, $\mathrm{n}^{\circ}$ 1, p. 177 - 182.

S. Prajna A. P., Parrilo P. A. (2002, dec.). Introducing sostools: A general purpose sum of squares programming solver. In The 41st ieee conference on decision and control, vol. 1, p. $741-746$.

Willems J. (1972). Dissipative dynamical systems. part i: general theory. Archive for Rational Mechanics and Analysis, vol. 45, n 5, p. 321-351. 